\title{
A randomized controlled study and evaluation of children with cerebral palsy by mind acupuncture
}

\author{
Liu $Z^{1 *}{ }^{*}$, Qi YC ${ }^{2}$, Yong $Z^{1}$, Ma MM' ${ }^{1}$, Qian XG ${ }^{1}$ and Fu WJ ${ }^{1}$ \\ ${ }^{1}$ Cerebral Palsy Rehabilitation Center, Nanhai Hospital for Women and Children, Affiliated to Guangzhou University of Chinese Medicine 528200, P.R.China \\ ${ }^{2}$ Guangzhou Cancer Hospital Guangdong 528200, P.R.China
}

\begin{abstract}
Objective: To investigate the effects of clearing the Governor Vessel and refreshing the mind needling in neural development and remediation of children with cerebral palsy.

Methods: 200 cases of children with cerebral palsy were randomly divided into the treatment group $(\mathrm{n}=100)$ and the control group $(\mathrm{n}=100)$. The treatment group was given the combined therapy of acupuncture and rehabilitation training, and the chosen acupoints were 13 points of the Governor Vessel, Shenshu (BL 23), Taixi (KI 3), Yanglingquan (GB 34), Zusanli (ST 36) and Sanyinjiao (SP 6), and points of refreshing the mind were also selected, which included puncturing Shenting (GV 24) toward Qianding (GV 21), puncturing Qianding (GV 21) toward Baihui (GV 20), puncturing Baihui (GV 20) toward Naohu (GV 17) and Sishencong (Ex-HN 1). The control group was only treated with rehabilitation training. A contrastive analysis of the therapeutic effect of acupuncture combined with rehabilitation training and pure rehabilitation training was made after a treatment course of 3 months. The Gross Motor Function Measure (GMFM) and Beijing Gesell Developmental Scale were adopted to assess the neural development and rehabilitation outcomes of the two groups. In addition, skull CT/MRI was adopted to evaluate the plerosis of injured cerebral nerve after treatment.
\end{abstract}

Results: The total effective rate in treatment group was $87 \%$, significantly higher than the 55\% in the control group. The children's development quotient (DQ) tested by Gesell Developmental Scale and scores tested by GMFM in the treatment group was obviously higher than the control group ( $<<0.01)$. The improving and curing rates presented by skull CT/MRI in the treatment group were higher than the control group $(\mathrm{P}<0.01)$.

Conclusions: Clearing the Governor Vessel and refreshing the mind Needling could accelerate the recovery of injured brain nerve and the reconstruction of brain function. The acupuncture therapy could ameliorate both the motor development and cognitive development. On the other hand, the forward curative effect of acupuncture combined with rehabilitation training was significantly better than the pure rehabilitation training.

\section{Introduction}

The medical rehabilitation of cerebral palsy was thought to be a global medical problem. In America for more than 60 years, many rehabilitation-training methods were applied in $\mathrm{CP}$ rehabilitation, but most of the children could not be cured after long-term rehabilitation training. Children with severe cerebral palsy often required a lifelong rehabilitation. In order to explore the efficacy of clearing the Governor Vessel and refreshing the mind needling to the rehabilitation of CP, from January 2005 to December 2006, we did a randomized study to compare the effect of acupuncture combined with rehabilitation training and pure rehabilitation training.

\section{Clinical data}

\section{Selection of cases}

(1) Diagnosis and classification standards were according to the 2006 national conference of cerebral palsy [1]; (2) age of 1 to 7 years old; (3) excluding progressive diseases or degenerative diseases, such as inherited metabolic disorders, and excluding neurosurgical diseases, such as brain tumors and hydrocephalus; (4) without loss of sight or nerve deafness; (5) obtained informed consent of all children's parents to take part in the study.

\section{General data}

200 cases were randomly divided into two groups, 100 cases in each group. Among the 100 cases in treatment group, 69 cases were male, and 31 cases were female. 66 cases were at the age of $1 \sqcup 3$, and 34 among $3+\sqcup 7,78$ cases with spastic cerebral palsy, 10 with involuntary motion, 8 with hypotonia, and 4 with ataxia. According to the Gross Motor Function Classification System (GMFCS), 28 cases were diagnosed with mild cerebral palsy and 72 with severe cerebral palsy. Besides, 86 of the cases were accompanied with mental retardation. Skull CT or MRI examination showed that 89 of the cases were with encephalopathies of periventricular leukomalacia, leukodystrophy or the brain's frontal and temporal lobe cortex dysplasia. In the control group, 83 cases were male and 17 female. 72 cases were at the age of

Correspondence to: Liu ZH, Cerebral Palsy Rehabilitation Center of Nanhai Maternity and Children Hospital Affiliated to Guangzhou University of Chinese Medicine (Foshan 528200) Guicheng borough, Nanhai, Foshan city, Guangdong province, China. 528200. Tel: 0757-86232936; Email: Lzh1958424@163.com

Key words: acupuncture therapy, cerebral palsy, nervous development, neurorestoratolog

Received: January 08, 2018; Accepted: January 30, 2018; Published: February 03,2018 
$1 \sim 3$, and 28 among $3+\sim 7,71$ cases with spastic cerebral palsy, 14 with involuntary motion, 13 with hypotonia, and 2 with ataxia. 23 cases were diagnosed with mild cerebral palsy and 77 with severe cerebral palsy. 79 cases were accompanied with mental retardation. Skull CT or MRI examination show that 83 of the cases with encephalopathies of periventricular leukomalacia, leukodystrophy or the brain's frontal and temporal lobe cortex dysplasia. The general situations of two groups, such as condition and course of diseases, were not statistically significant $(\mathrm{P}>0.05)$.

\section{Therapeutic approaches}

\section{Rehabilitation training}

Physiotherapy (PT), occupational therapy (OT) and speech therapy (ST) were mainly selected for the rehabilitation training. The Bobath therapy was used to inhibit the abnormal posture, abnormal postural reflex and abnormal motion pattern and to facilitate the neck righting reflex, body righting reflex, equilibrium reaction and standing static and dynamic balance. OT was adopted to treat upper limb movement disorders or hand-eye coordination disorders. ST was used to treat speech developmental retardation or dysarthria. The children were given one-to-one rehabilitation training of $1 \sim 2$ hours per day, 90 days made up a course of treatment.

\section{Acupuncture therapy}

(1) Clearing the Governor Vessel Needling: 13 points of the Governor Vessel, Shenshu (BL 23), Taixi (KI 3), Yanglingquan (GB 34), Zusanli (ST 36) and Sanyinjiao (SP 6), with mild reinforcing-reducing method was applied, needles were not retained in children under 3 or with delicate health, but for 30 minutes to those over the age of 3 .

(2) Refreshing the Mind Needling: puncturing Shenting (GV 24) toward Qianding (GV 21), puncturing Qianding (GV 21) toward Baihui (GV 20), puncturing Baihui (GV 20) toward Naohu (GV 17) and Sishencong (Ex-HN 1), assisted by scalp motor area, scalp foot motor sensory area and balance area, with speech areas 1,2 and 3 adopted for language disorder cases. Acupuncture methods: inserting rapidly, retaining needles for 4 hours and twirling 3 times for $1 \sim 3$ minutes at the frequency of 200 times per minute at interval.

Course of Treatment: Qod for 10 times, 30 times make up a course, with a rest of 20 days after every 10 treatments.

The control group was only given rehabilitation training. The treatment group was given the acupuncture combined with rehabilitation training.

\section{Rehabilitation assessment indexes and therapeutic ef- fect evaluation criteria}

\section{Rehabilitation assessment indexes}

(1) Indexes of Neurodevelopment: The Beijing Gesell Developmental Scale [2] was used to evaluate the children's development quotients (DQ) of social adaptation, personal communication, speech, gross motor movement and fine motor movement. The Gross Motor Function Measure (GMFM) ${ }^{[3]}$ was applied to assess the gross motor development before and after treatment. (2) Indexes of Nerve Repair: Skull CT/MRI was used to observe the recovery of neural structure after the treatment.

\section{Therapeutic effect evaluation criteria}

Markedly Effective: The DQ increased by 15, total score of GMFM increased by a standard deviation, and skull CT/MRI re-scanning showed an improved or recovery of brain cortex dysplasia and periventricular leukomalacia; [3]

Effective: The DQ increased by 10, total score of GMFM increased by 10 , and skull CT/MRI re-scanning showed an improvement of brain hypogenesis;

Ineffective: The DQ increased by less than 10, and skull CT/MRI re-scanning showed no improvement.

\section{Statistical methods}

The SPSS12.O statistical package was adopted, with $x^{2} \pm s$ to express the measurement data, $\mathrm{x}^{2}$ test to compare the rates and variance analysis to compare means in groups.

\section{Results}

\section{Rehabilitation effect comparison between the two groups}

For 100 cases in the treatment group, 39 cases were markedly effective (39\%), 48 cases were effective ( $48 \%), 13$ cases were ineffective (13\%). The overall response rate was $87 \%$. By contrast, for 100 cases in the control group, 23 cases were markedly effective (39\%), 32 cases were effective $(48 \%), 45$ cases were ineffective $(45 \%)$. The overall response rate was $55 \%$.The total effective rate in the treatment group was significantly higher than the control group $\left(\mathrm{x}^{2}=12.546, \mathrm{P}<0.001\right)$. At 1-year follow-up for analysis of long-term effect, 8 of treatment group and 10 of control group lost visit. In the treatment group, 47 cases were markedly effective $(51.09 \%), 37$ cases were effective ( $40.21 \%), 8$ cases were ineffective $(8.7 \%)$. The overall response rate was $91.3 \%$. The corresponding dates of control group were $18(20.00 \%)$, $21(23.33 \%), 51(56.67 \%)$, with overall response rate of $43.33 \%$. The overall response rate of treatment group was obviously higher than control group $\left(\mathrm{x}^{2}=47.786, \mathrm{P}<0.001\right)$, which showed a superiority in long-term rehabilitation effect (Tables 1-4).

\section{Manifestations of the skull CT/MRI after treatment}

In the treatment group, 89 cases were with abnormal CT/MRI manifestations of encephalopathies of periventricular leukomalacia, leukodystrophy or the brain's frontal and temporal lobe cortex dysplasia before treatment, and of the 89 cases, 29 cases (12 with periventricular

Table 1. GMFM scores comparison of the two groups before and after treatment $(\bar{x} \pm s)$

\begin{tabular}{|c|c|c|c|c|}
\hline \multirow{2}{*}{ Group } & \multirow{N}{*}{$\boldsymbol{N}$} & \multicolumn{3}{|c|}{ GMFM scores } \\
\cline { 3 - 5 } & & Pre-treatment & Post-treatment & Follow-up \\
\hline Treatment & 100 & $34.29 \pm 21.82$ & $86.16 \pm 18.94^{*}$ & $93.87 \pm 17.84^{* \#}$ \\
\hline Control & 100 & $36.33 \pm 19.75$ & $69.42 \pm 25.23^{*}$ & $76.63 \pm 22.85^{* \#}$ \\
\hline$t$ & - & -0.817 & -2.449 & -2.673 \\
\hline$P$ & - & 0.432 & 0.016 & 0.013 \\
\hline
\end{tabular}

Note: The "**" stands for " $\mathrm{P}<0.01$ " when comparing with pre-treatment interior-group, and "\#" stands for " $\mathrm{P}<0.01$ " when comparing with post-treatment interior-group. 8 cases in treatment group and 10 in control group lost follow-up visit.

Table 2. Gross Motor DQ comparison of the two groups before and after treatment $(\bar{x} \pm s)$

\begin{tabular}{|c|c|c|c|c|}
\hline \multirow{2}{*}{ Group } & \multirow{N}{*}{} & \multicolumn{3}{|c|}{ Gross Motor DQ } \\
\cline { 2 - 5 } & & Pre-treatment & Post-treatment & Follow-up \\
\hline Treatment & 100 & $35.89 \pm 14.69$ & $68.89 \pm 13.76^{*}$ & $86.15 \pm 13.84^{* \#}$ \\
\hline Control & 100 & $39.35 \pm 12.68$ & $48.84 \pm 16.85^{*}$ & $56.60 \pm 14.55^{* \#}$ \\
\hline$t$ & - & 0.716 & -2.808 & -4.257 \\
\hline$P$ & - & 0.439 & 0.012 & 0.0011 \\
\hline
\end{tabular}

Note: The "*" stands for " $\mathrm{P}<0.01$ " when comparing with pre-treatment interior-group, and "\#” stands for " $\mathrm{P}<0.01$ " when comparing with post-treatment interior-group. 8 cases in treatment group and 10 in control group lost follow-up visit. 
Table 3. Fine Motor DQ comparison of the two groups before and after treatment $(\bar{x} \pm s)$

\begin{tabular}{|c|c|c|c|c|}
\hline \multirow{2}{*}{ Group } & \multirow{N}{*}{$\boldsymbol{N}$} & \multicolumn{3}{|c|}{ Gross Motor DQ } \\
\cline { 2 - 5 } & & Pre-treatment & Post-treatment & Follow-up \\
\hline Treatment & 100 & $39.09 \pm 19.42$ & $69.71 \pm 12.54^{*}$ & $79.65 \pm 13.56^{* \#}$ \\
\hline Control & 100 & $38.18 \pm 21.29$ & $51.43 \pm 13.69^{*}$ & $54.79 \pm 12.61^{* \#}$ \\
\hline$t$ & - & -1.134 & -2.126 & -2.967 \\
\hline$P$ & - & 0.262 & 0.022 & 0.007 \\
\hline
\end{tabular}

Note: The “*” stands for " $\mathrm{P}<0.01$ " when comparing with pre-treatment interior-group, and "\#" stands for " $\mathrm{P}<0.01$ " when comparing with post-treatment interior-group. 8 cases in treatment group and 10 in control group lost follow-up visit.

Table 4. Social Adaptation DQ comparison of the two groups before and after treatment $(\bar{x} \pm s)$

\begin{tabular}{|c|c|c|c|c|}
\hline \multirow{2}{*}{ Group } & \multirow{2}{*}{$\boldsymbol{N}$} & \multicolumn{3}{|c|}{ Social Adaptation DQ } \\
\cline { 3 - 5 } & & Pre-treatment & Post-treatment & Follow-up \\
\hline Treatment & 100 & $35.27 \pm 22.76$ & $63.69 \pm 13.63^{*}$ & $78.76 \pm 12.65^{* \#}$ \\
\hline Control & 100 & $35.35 \pm 23.93$ & $51.22 \pm 15.68^{*}$ & $55.81 \pm 15.49^{* \#}$ \\
\hline$Z$ & - & -0.141 & -2.127 & -2.818 \\
\hline$P$ & - & 0.898 & 0.024 & 0.014 \\
\hline
\end{tabular}

Note: The “*” stands for "P $<0.01$ " when compared with pre-treatment interior-group, and "\#" stands for " $\mathrm{P}<0.01$ " when compared with post-treatment interior-group. 8 cases in treatment group and 10 in control group lost follow-up visit.

leukomalacia and leukodystrophy, 17 with cortex dysplasia) returned to normal after treatment, 24 cases got improvement, with a total amelioration rate of $59.55 \%$ (53/89). In contrast, of the 83 cases with abnormal CT/MRI manifestations in the control group, only 6 cases got fully recovery and 5 got improvement, with a total amelioration rate of $13.25 \%(11 / 83) . \mathrm{x}^{2}$ test and T-test showed that the differences inter-group were statistically significant $\left(\mathrm{x}^{2}=11.257, \mathrm{P}<0.001\right)$, which approved that clearing the Governor Vessel and refreshing the mind Needling could accelerate the recovery of injured brain nerve and the reconstruction of brain function.

The analysis of the above statistics showed that clearing the Governor Vessel and refreshing the mind Needling combined with rehabilitation had superiority in ameliorating both the motor development and cognitive development compared to rehabilitation training alone.

\section{Discussions}

Children cerebral palsy belonged to "five retardations" in traditional Chinese medicine, which originated from congenital deficiency. Zhang Shi Yi Tong ("Zhang medical pass") stated that the causes of "five retardations" were asthenia of parents' essence and blood and Kidney Qi deficiency. According to theory of traditional Chinese medicine, Kidney was the Zang generating Marrow, and adequate Kidney essence was the basic of producing Marrow. The Kidney essence included congenital essence inherited from parents and acquired essence transformed by Spleen. The Marrow was transferred by the Governor Vessel to fill up the brain. On the other hand, the Governor Vessel was the sea of Yang meridians, and was thought to be full of Yang-qi. The Sheng Qi Tong Tian Lun Pian of Plain Questions ("Su asked • angry universe theory article") stated that "Yang-qi acts to nourish the spirit and tendons". Gu Kong Lun of Plain Question ("Su asked • bone empty on") stated that the disorder of the Governor Vessel characterized by stiffness and opisthotonus. Besides, the brain was the house of mentality and the Mind was the manifestation of brain function. So we came to the conclusion that the essential therapeutic principle for cerebral palsy is to regulate the brain, refresh the mind, strengthen the Spleen, and tonify the Kidney. The results of the study showed that clearing the Governor Vessel and refreshing the mind needling combined with rehabilitation training got better effect in both the motor improvement and cognitive improvement when compared to the pure rehabilitation training.

Report showed that needling Zusanli (ST 36) and Sanyinjiao (SP 6) could stimulate areas of postcentral gyrus, inferior frontal gyrus, insular lobe, inferior parietal lobule, culmen of cerebellum and middle temporal gyrus of the brain [4]. It's also reported that needling Zusanli (ST 36) and Futu (ST 32) could increase the average BoLD signal intensity of hypothalamus, hippocampal gyrus and frontal gyrus through fMRI; needling Yanglingquan (GB 34) could increase the average BoLD signal intensity in the occipital lobe, forehead, temporal lobe, basal ganglia and pons [5]; needling motor area in the scalp of the stroke patients could improve the local cerebral blood flow perfusion and brain function confirmed by SPECT examination [6]; needling Baihui (GV 20) and Qubin (GB 7) could elevate or reduce the glucose metabolism of the bilateral brain motor area and thus induce or excite nerve tissue of some motor-related areas, compensate or assist the impaired neural network, and improve the reconstruction of motor function [7]. Another report showed that needling Zusanli (ST 36) and Sanyinjiao (SP 6) could improve the local cerebral blood flow perfusion and brain function of 17 paralytic patients confirmed by SPECT examination $[8,9]$. Actually, we obtained the similar results with the above reports. The clearing the Governor Vessel and refreshing the mind Needling could accelerate the recovery of injured brain nerve and the reconstruction of brain function. Acupuncture therapy improved the motor function and got remarkable effect in accelerating the cognitive development. The most remarkable change was the imaging results of skull CT/MRI scanning in the treatment group, with the recovery rate of brain hypogenesis in the acupuncture group was $59.55 \%$ (53/89), which was much higher than the $13.25 \%(11 / 83)$ in the non-acupuncture group.

The research findings through imageology (CT/MRI) suggested that needling techniques of clearing the Governor Vessel and refreshing the mind could promote the recovery of brain nerves and brain functional reconstruction of kids with cerebral palsy, so as to enhance the motor function and cognitive ability. Cases in the acupuncture group got significant improvement in DQ of gross motor movement, fine motor movement and social adaptation after the treatment, which indicated that acupuncture therapy had multiple repair actions in treatment of cerebral palsy. It was quite possible that acupuncture therapy could accelerate the neurological cells to grow in the direction of helping the axoneure to actualize normal integration and regulation by promoting the growth of neurological cells [10], with the benefit of that the normal axoneure would compensate the function of impaired axoneure gradually.

\section{Acknowledgement}

This work is supported by National Science Foundation of China. (NO.3074007)

\section{References}

1. Zi WC, Zhi ZM (2006) The $2^{\text {nd }}$ Children Rehabilitation Conference (Chinese Association of Rehabilitation Medicine), the $9^{\text {th }}$ children Cerebral Palsy Rehabilitation Conference (China Association of Rehabilitation of Disabled Persons) And International Communication Conference. Journal of Clinical Pediatrics 21: 1742.

2. Liu ZH (2005) Family Rehabilitation of Children Cerebral Palsy [M] (China). Hong Kong: Hong Kong Pharmaceutical Press. 112-119.

3. Liu ZH, Ma MM, Pei PG (2004) Exploration of Rehabilitation Modality for Cerebral Palsy in China. Clinical Journal of Traditional Chinese Medicine 16: 414-416.

4. Song FH, Shi ZZ, Liu H (2006) MRI Investigation of Brain Reaction by Acupuncture Chinese Journal of Integrated Traditional and Western Medicine 26: 965-968. 
5. Cheng Z, Jia WZ, Min C (2005) MRI Manifestation on Needling Acupoints and Cerebral Cortex. Chinese Journal of Radiology 39: 252-253.

6. YANG OG, Shao ZW, Fan W (2005) Effect of Different Electric Stimulation Frequencies on Cerebral Blood Flow Peralsion and Brain Function of Cases with Cerebral Apoplexy. Chinese Acupuncture \& Moxibustion 25: 776-778.

7. Xian S, Fang Z, Jia TH (2005) Effect of Needling Scalp Points on Stroke Patients' Glucose Metabolism in Motor, Domain of the Brain. Acupuncture Research 30: 167-170.
8. Ai SJ, Wei Y, Zhao WK, et al. Effect of Acupuncture on the Blood Stream Perfusion and Functional Activities of Brain in Paitents with Severe Brain Injury. Chinese Journal of Clinical Rehabilitation 8: 5312-5313.

9. Yong H, Jun CQ, Yan ZQ (2009) Comparison of fMRI Between Needling Waiguan (SJ5) And Combined S J5 and Neiguan (PC6), Memoir of International Conference on Traditional Medicine 53-58.

10. Qin WY, Wen WH, Ru J (2005) Effects of Acupunture on GFAP Expression in Asphyxial Cerebral Paralysis Rats. Chinese Journal of Rehabilitation Medicine 20: 423-426.

Copyright: C2018 Liu ZH. This is an open-access article distributed under the terms of the Creative Commons Attribution License, which permits unrestricted use, distribution, and reproduction in any medium, provided the original author and source are credited. 